\title{
Hiperglucemia en la urgencia pediátrica. No todo es diabetes
}

\author{
Hyperglycemia in the pediatric emergency. \\ Not everything is diabetes
}

\author{
Dr. Manuel D. Bilkis ${ }^{a}$
}

\section{RESUMEN}

La hiperglucemia es un hallazgo poco frecuente en la urgencia pediátrica.

La hiperglucemia, glucemia $\geq 126 \mathrm{mg} / \mathrm{dl}$, puede corresponder a un cuadro de diabetes mellitus tipo 1; el hallazgo de una hiperglucemia casual en un paciente obeso y con diabetes tipo 2; la hiperglucemia sin descompensación de una diabetes monogénica o ser una hiperglucemia por estrés.

Estas últimas suelen ser no cetósicas, limitadas a la enfermedad aguda $\mathrm{y}$, generalmente, no desarrollan diabetes en el seguimiento posterior. En un análisis, hayamos solo un 2,9\% de casos (8/270 niños) con hiperglucemia por estrés que desarrollaron diabetes mellitus en el seguimiento posterior.

El uso de insulina en los casos más graves mejora la evolución y disminuye la morbimortalidad. Existe una superposición de cuadros intermedios en la presentación de la hiperglucemia, que requiere el auxilio del especialista para desentrañar el cuadro subyacente en el seguimiento posterior.

Palabras clave: hiperglucemia, estrés, emergencia pediátrica, glucosa, insulina.

\begin{abstract}
Hyperglycemia is a rare finding in pediatric emergency.

Hyperglycemia as pediatric emergency presentation (blood glucose $\geq 126 \mathrm{mg} / \mathrm{dl}$ ), may correspond to a diabetes mellitus type 1 , the finding of a casual hyperglycemia in an obese patient and type 2 diabetes, hyperglycemia without decompensation of the monogenic diabetes or stress hyperglycemia. The latter are often not ketosis, limited to acute illness and usually do not develop diabetes at follow-up. We found only $2.9 \%$ of patients ( $8 / 270$ children) with stress hyperglycemia who developed diabetes mellitus at follow-up. The use of insulin in the most severe cases improves the evolution and decreases morbidity. There is an overlap of intermediate states in the presentation of hyperglycemia requiring specialist help to unravel the underlying state in the follow-up. Key words: hyperglycemia, stress, pediatric emergency, glucose, insulin.
\end{abstract}

Dr. Manuel D. Bilkis: mbilkis@gmail.com

Conflicto de intereses: Ninguno que declarar.

Recibido: 28-4-2014 Aceptado: 11-9-2014 de emergencia pediátrica (DEP). En el estudio multicéntrico PECARN, las causas endócrinas, metabólicas y nutricionales fueron todas juntas la novena causa de consulta en el DEP. Sin embargo, su presencia aumentó la posibilidad de internación entre 6 y 7 veces. ${ }^{1}$ En nuestro medio, solo hallamos un $0,2 \%$ ( $3 / 1435$ casos) de consultas que se correspondieron a diabetes en el DEP. ${ }^{2}$ La hiperglucemia no necesariamente significa diabetes. Sament y Schwartz (1957) diferenciaron la hiperglucemia sin cetosis y la cetoacidosis diabética. ${ }^{3}$

Entre la etiología de la hiperglucemia sin cetosis por estrés, se destacan infecciones y otras alteraciones (hipermetabolismo, hipercatabolismo, aumento de movilización y oxidación de grasas y resistencia a la glucosa e insulina en sépticos y traumatizados [estresados] y que responden al aumento de hormonas contrarreguladoras [hormona de crecimiento, glucagón, cortisol y noradrenalina]). ${ }^{4}$ También las citoquinas inflamatorias promueven la lipólisis y la proteólisis y la gluconeogénesis. La resistencia a la insulina hepática prevalece y hay abundante producción endógena de glucosa a pesar de los niveles elevados de glucosa. La captación estimulada por insulina de la glucosa por los tejidos periféricos, tales como el esquelético, muscular, adiposo y cardíaco, se reduce.

La hiperglucemia por estrés en el paciente crítico es una respuesta adaptativa. También ha sido reconocida como signo de mal pronóstico. ${ }^{5,6}$ Por lo general, la hiperglucemia por estrés es definida como una hiperglucemia que se 
soluciona de manera espontánea después de que se resuelve la enfermedad aguda. El término, generalmente, refiere a pacientes sin diabetes conocida; sin embargo, pacientes con diabetes pueden desarrollar hiperglucemia por estrés, un factor que, a veces, es pasado por alto en estudios de pacientes internados con y sin diabetes. En varios estudios, se sugiere que la hiperglucemia por estrés aumenta el riesgo de morbimortalidad, sobre todo en los pacientes sin diabetes. ${ }^{7}$ Así, podríamos pensar la hiperglucemia por estrés como un epifenómeno que se puede presentar en pacientes con o sin diabetes, o también un grupo intermedio sin diabetes actual, pero en los que desconocemos su futuro.

La hiperglucemia por estrés depende de la enfermedad aguda (activación del eje hipotalámico-hipofisario-adrenal, liberación de catecolaminas, citoquinas proinflamatorias y/o lipotoxicidad), de las características del paciente (reserva pancreática, autoanticuerpos antiinsulina o antiislotes de células B de Langerhans o resistencia insulínica periférica) y/o de la terapéutica empleada (corticoides, L-asparaginasa, nutrición parenteral total, vasopresores, etc.). ${ }^{7}$

\section{Hiperglucemia en el \\ Departamento de Emergencias pediátrico}

Aşılıoğlu N. y col. consideran hiperglucemia cuando presenta valores de glucemia en ayunas mayores de $150 \mathrm{mg} / \mathrm{dl}(8,3 \mathrm{mmol} / \mathrm{L}){ }^{6}$ Estos valores de corte de hiperglucemia no se corresponden con los de la American Diabetes Association, que consideran hiperglucemia a valores de glucemia $\geq 126 \mathrm{mg} / \mathrm{dl}(7 \mathrm{mmol} / \mathrm{L})$ con ocho horas de ayuno previo. ${ }^{30}$

Weiss y col. ${ }^{9}$ hallaron hiperglucemia en alrededor del $5 \%$ de los pacientes que concurren al DEP y puede ascender hasta un $25-60 \%$ de casos en enfermedades graves. Cuando los valores de glucemia se hallan por encima de los $300 \mathrm{mg}$ / dl (16,7 mmol/L), se habla de hiperglucemia por estrés extrema, y Weiss y col. la encontraron en solo $0,13 \%$ (75/55 120 casos) de niños que concurrieron a un DEP en un período de 6 años. ${ }^{9}$

Cuando se presenta un niño con hiperglucemia, la etiología para pensar sería la siguiente:

a) Primero, por frecuencia en diabetes mellitus tipo 1, por lo que debemos interrogar sobre antecedentes, en los casos de diabetes ya conocidas, o la presencia de poliuria, polidipsia y pérdida de peso. Con cetosis grave: anorexia marcada, dolor abdominal y vómitos. Deshidratación y / o choque hipovolémico en cetoacidosis moderada o grave. El sensorio puede ser alternante y en formas graves de cetoacidosis, obnubilación y/o coma. ${ }^{10}$ Se puede presentar con descompensación metabólica (cetoacidosis diabética).

b) También en casos de obesidad en adolescentes, sin cetoacidosis, puede ser la hiperglucemia en el DEP el hallazgo de una diabetes mellitus tipo 2.

c) En tercer lugar, el avance en el conocimiento de los trastornos genéticos asociados a enfermedad hace que hoy en día podamos individualizar un grupo de diabéticos cuyas características son las de presentar una diabetes de tipo 2 (con escasa insulinorresistencia, pero con alteración en la función de la célula beta) y que no requiere insulinización inmediata al diagnóstico. Siempre se presenta en edades inferiores a los 25 años, sin obesidad y con un patrón de herencia autosómica dominante. Se la tipifica como diabetes tipo MODY (mature onset diabetes in the young) o diabetes del adulto de comienzo infantil. Si bien la diabetes tipo $1 \mathrm{y}$, sobre todo, la de tipo 2 tienen una base, al menos parcialmente, genética $y$, en este sentido, se pueden presentar a menudo en varios individuos de la misma familia (aunque sin un patrón mendeliano establecido), existe, como indicábamos anteriormente, una diabetes que se diagnostica en la infancia, adolescencia o adultos jóvenes, con herencia autosómica dominante (o sea, presencia de diabetes en todas las generaciones y en el $50 \%$ de los hijos) y que clínicamente se comporta como una diabetes tipo 2 , sin cetoacidosis de comienzo. Su origen se ha asociado con trastornos en diferentes genes que codifican para proteínas implicadas en la función de la célula beta (glucokinasa, factor nuclear del hepatocito [(HNF) 1 alfa, HNF 4 alfa, HNF 1 beta, factor promotor de insulina 1]. Desde el punto de vista clínico, sus características varían en función del gen alterado: van desde cuadros infantiles de hiperglucemias permanentes pero leves o moderadas y con buen pronóstico clínico (el déficit de glucokinasa o MODY 2) a cuadros con hiperglucemias mantenidas que se acompañan, si existe un mal control glucémico, de complicaciones crónicas graves y precoces (MODY 3 o déficit de factor HNF 1a). Al momento del diagnóstico, la diabetes MODY 
puede confundirse con una diabetes mellitus tipo 1, pero no se acompaña de cetoacidosis, no tiene autoanticuerpos circulantes y tiene una herencia dominante característica. ${ }^{11} \mathrm{Tal}$ Oron y col. (2011) describen dos casos de hiperglucemia por estrés en el DEP, una en un neonato de 5 días y otra en un niño de 2 años, ambos con presencia confirmada de alteración monogenética heterocigota de la glucoquinasa (MODY 2). ${ }^{12}$ La presencia elevada de hemoglobina glicosilada en ambos casos, combinada con diabetes gestacional en la madre del recién nacido y antecedentes familiares de diabetes tipo 2 en el otro caso, hizo sospechar la posibilidad de encontrar diabetes monogénica en estos casos de hiperglucemia por estrés y fiebre. ${ }^{12}$

En un estudio de cohorte de 40 lactantes menores de un año con diagnóstico de diabetes mellitus, el 85\% de los casos en menores de 6 meses y el $55 \%$ de los diagnosticados entre los 6 meses y el año fueron monogénicas. ${ }^{13}$

La diabetes mellitus es un trastorno etiológicamente heterogéneo. Las formas monogénicas de la diabetes mellitus cubren un heterogéneo grupo de diabetes que son causadas uniformemente por una sola mutación del gen y se caracterizan por la alteración de la secreción de insulina de las células beta pancreáticas. Se estima que representan hasta el 5\% de todos los casos de la diabetes mellitus, que a menudo no son diagnosticados o son mal clasificados como diabetes tipo 1 o 2 . Sin embargo, el diagnóstico preciso es importante por el pronóstico, el riesgo de la familia y las implicaciones especiales para el tratamiento ya que pueden responder a los hipoglucemiantes orales. El conocimiento de las características clínicas típicas, como modo de herencia, la edad de diagnóstico y la alteración de la secreción de insulina, así como la genética, establece el diagnóstico de MODY, diabetes mitocondrial, una forma hereditaria de diabetes asociada con pérdida auditiva y deterioro progresivo de la función pancreática y la diabetes neonatal (diabetes monogénica en menores de 6 meses). ${ }^{14}$

d) En cuarto lugar, está la hiperglucemia por estrés secundaria a enfermedades agudas que generan respuestas adaptativas al estrés, que incluyen hiperglucemias. También en pacientes que tienen enfermedades agudas, el uso de drogas que estimulan la hiperglucemia puede ocasionar este efecto. Fuera de la diabetes, la hiperglucemia puede ser de causa exógena (ejemplo: uso de corticoides y L-asparaginasa en la inducción de la leucemia aguda) ${ }^{4}$ o endógena por estrés y está dada por la elevación del cortisol, glucagón, hormona de crecimiento, catecolaminas y varias citocinas que estimulan la glucogenólisis y la gluconeogénesis. La glucemia retorna a la normalidad cuando el factor que produjo el estrés desaparece. ${ }^{8}$

\section{Hiperglucemia por estrés}

¿Mejora la evolución el uso de insulina para la hiperglucemia por estrés?

Esta es una pregunta aún sin respuesta. ${ }^{4,9,15}$ L. Rabinowitz y col. ${ }^{15}$ lograron normalizar la glucemia solo con la hidratación y sin el uso de insulina en niños con gastroenteritis e hiperglucemia. Weiss y col. ${ }^{9}$ revisaron 75 niños con hiperglucemia por estrés extrema $\mathrm{y}$, solo en 8 casos, recibieron tratamiento las hiperglucemias: en dos casos, se les suspendió el aporte de dextrosa en los sueros endovenosos y, en 6 casos, se les suministró insulina; 3 de los 6 casos desarrollaron hipoglucemia luego de recibir insulina.

En series de casos de hiperglucemia, leucemia e inducción de glucocorticoides y L-asparaginasa, la mayoría de los pacientes recibieron insulina por períodos cortos $\mathrm{y}$, en algunos casos, presentaron hipoglucemia postinsulina. ${ }^{16,17}$

D. Macrae y col. ${ }^{18}$ llevaron a cabo un estudio multicéntrico en niños que recibieron ventilación mecánica en terapia intensiva y en los cuales se aleatorizó el nivel de glucosa para recibir insulina: mayor de 126 o $216 \mathrm{mg} /$ dl. La morbimortalidad no demostró diferencias entre ambos grupos; sin embargo, aumentaron los episodios de hipoglucemia en el grupo que corregía la hiperglucemia con valores más bajos (126 mg/ dl). En grupos de niños con quemaduras o cirugía cardiovascular con hiperglucemia por estrés y necesidad de terapia intensiva, el control estricto de la hiperglucemia con insulina intravenosa mejoró la morbimortalidad. ${ }^{19}$

B. Poddar ${ }^{5}$ revisó el tratamiento con insulina en niños graves con hiperglucemia y concluyó que el monitoreo de la glucemia y la corrección de la hiperglucemia es crucial, ya que disminuye la morbimortalidad de la enfermedad de base. Si se busca mantener niveles normales de glucosa con aporte de insulina, se corre el riesgo de provocar hipoglucemia. Para evitar la hipoglucemia, es necesario un adecuado aporte nutricional y un monitoreo continuo. ${ }^{5}$ La corrección de la 
hiperglucemia por estrés con insulina depende de la gravedad del cuadro y puede dar lugar a episodios de hipoglucemia.

¿La hiperglucemia por estrés predispone a la diabetes?

En una revisión en adultos, K. Dungan y col. $(2009)^{7}$ revisaron 7 series de pacientes con hiperglucemia por estrés en pacientes hospitalizados, de los cuales entre un $27 \%$ y un $42 \%$ presentaron alteración en la prueba de tolerancia a la glucosa y, en el seguimiento posterior, un 10\%-60\% desarrollaron diabetes mellitus en un plazo menor de un año. En niños, sería distinto.

Para responder a esta pregunta, se realizó una búsqueda en Medline (pubmed.gov) con los términos MESH: hyperglycemia and children and stress. Se hallaron 132 artículos, de los que se seleccionaron 9 series de casos de hiperglucemia por estrés con seguimiento posterior, que intentan, por distintos métodos, establecer la predisposición a desarrollar diabetes mellitus (Tabla 1). Haciendo un análisis de estas 9 series de niños con hiperglucemia por estrés, se estudiaron globalmente 270 casos, de los cuales solo 8 niños $(2,9 \%)$ desarrollaron diabetes mellitus en el seguimiento posterior.

Podríamos responder a la pregunta inicial diciendo que la hiperglucemia por estrés, generalmente, es un cuadro adaptativo que se observa en la fase aguda de algunas enfermedades y, en la gran mayoría de los casos, no deriva en diabetes.

Sin embargo, algunos autores describen casos puntuales de hiperglucemia por estrés y diabetes. ${ }^{12,20}$ En uno de los casos con hiperglucemia por estrés, ${ }^{4}$ una adolescente con

TABLA 1. Trabajos de series de casos con hiperglucemia por estrés y estudio de desarrollo de diabetes mellitus

\begin{tabular}{|c|c|c|c|c|}
\hline & Autores & $\begin{array}{c}\text { Número de casos } \\
\text { Edad: mediana (rango) }\end{array}$ & Métodos de estudio & $\begin{array}{c}\text { Desarrollo de } \\
\text { diabetes mellitus (DM) }\end{array}$ \\
\hline 1) & $\begin{array}{l}\text { P. Vardi y col., } \\
1990^{20}\end{array}$ & $\begin{array}{l}12 \text { niños: } 7,2 \text { años } \\
\text { ( } 2 \text { meses-14 años). }\end{array}$ & $\begin{array}{l}\text { Anticuerpos antiinsulina, } \\
\text { anticuerpos antiislotes, } \\
\text { prueba de tolerancia a glucosa } \\
\text { endovenosa. }\end{array}$ & $\begin{array}{c}4 / 12(33 \%) \text { DM } \\
\text { en menos de } 1 \text { año. } \\
5 \text { pacientes con pruebas } \\
\text { patológicas. }\end{array}$ \\
\hline 2) & $\begin{array}{l}\text { N. Shehadeh y col., } \\
1997^{21}\end{array}$ & $\begin{array}{l}36 \text { niños: 6,2 años } \\
\text { (1-17 años). }\end{array}$ & $\begin{array}{l}\text { Anticuerpos antiislotes, } \\
\text { anticuerpos antiinsulina, } \\
\text { prueba de tolerancia a } \\
\text { glucosa endovenosa. }\end{array}$ & $\begin{array}{l}\text { Ninguno desarrolló DM } \\
\text { (seguimiento promedio: 3,2 años). } \\
\text { Algunos presentaron alguna } \\
\text { alteración en las pruebas } \\
\text { complementarias. }\end{array}$ \\
\hline 3) & $\begin{array}{l}\text { D. Bhisitkul y col., } \\
1996^{22}\end{array}$ & $\begin{array}{l}30 \text { niños: } 2 \text { años } \\
\text { (1 mes-12 años). }\end{array}$ & $\begin{array}{l}\text { Anticuerpos antiislotes, } \\
\text { anticuerpos antiinsulina, } \\
\text { ácido glutámico decarboxilasa, } \\
\text { gen DQB1. }\end{array}$ & $\begin{array}{l}\text { Ninguno desarrolló DM. } \\
\text { Algunos presentaron alguna } \\
\text { alteración en las pruebas } \\
\text { Complementarias. }\end{array}$ \\
\hline 4) & $\begin{array}{l}\text { G. Valerio y col., } \\
2001^{23}\end{array}$ & 41 niños. & Ninguno. & $\begin{array}{l}\text { Ninguno desarrolló DM; } \\
\text { seguimiento: } 3,5 \text { años (2-4 años). }\end{array}$ \\
\hline 5) & $\begin{array}{l}\text { L. Rabinowitz y col., } \\
1984^{15}\end{array}$ & $\begin{array}{l}15 \text { niños con gastroenteritis } \\
\text { y deshidratación: } \\
8,6 \text { meses ( } 6 \text { meses-2 años). }\end{array}$ & $\begin{array}{l}\text { Insulina, ácidos grasos libres, } \\
\text { glucagón, cortisol, hormona } \\
\text { de crecimiento, péptido C. }\end{array}$ & $\begin{array}{l}\text { Disminución de la insulina y } \\
\text { hormonas contrarreguladoras } \\
\text { con la hidratación. }\end{array}$ \\
\hline 6) & $\begin{array}{l}\text { S. Weiss y col., } \\
2010^{9}\end{array}$ & $\begin{array}{c}72 \text { niños con hiperglucemia } \\
\text { por estrés extrema: } \\
8,8 \text { años }(2,6-13,9 \text { años }) .\end{array}$ & Glucemias seriadas. & $\begin{array}{l}\text { Un } 25 \% \text { desarrolló hiperglucemia } \\
\text { transitoria; } 1 \text { paciente desarrolló } \\
\text { hiperglucemia por corticoide. }\end{array}$ \\
\hline 7) & $\begin{array}{l}\text { M. Bordbar y col., } \\
2012^{24}\end{array}$ & $\begin{array}{l}39 \text { niños: } 26 \text { meses } \\
\text { (1 mes-4 años). }\end{array}$ & $\begin{array}{l}\text { Insulina, hormonas tiroideas, } \\
\text { cortisol, hormona de crecimiento, } \\
\text { glucemia en ayunas. }\end{array}$ & $\begin{array}{l}\text { Ninguno desarrolló DM } \\
\text { (seguimiento de } 2 \text { años). }\end{array}$ \\
\hline 8) & $\begin{array}{l}\text { A. Rosenbloom y col., } \\
1982^{25}\end{array}$ & $\begin{array}{c}37 \text { niños: } 3 \text { con } \\
\text { hiperglucemia por estrés, } \\
13 \text { con hipoglucemia y } \\
21 \text { con glucosuria. }\end{array}$ & $\begin{array}{l}\text { Prueba de tolerancia oral } \\
\text { a la glucosa. }\end{array}$ & $\begin{array}{l}3 \text { niños desarrollaron DM. } \\
\text { Todos con prueba anormal } \\
\text { de tolerancia a la glucosa. }\end{array}$ \\
\hline 9) & $\begin{array}{l}\text { E. Saz y col., } \\
2011^{26}\end{array}$ & $\begin{array}{l}22 \text { niños: } 4,4 \text { años } \\
\text { (3 meses-8 años). }\end{array}$ & $\begin{array}{l}\text { Prueba de tolerancia oral } \\
\text { a la glucosa, anticuerpos } \\
\text { antiinsulina. }\end{array}$ & $\begin{array}{c}\text { Ninguno } \\
\text { desarrolló DM. }\end{array}$ \\
\hline
\end{tabular}


hiperglucemia por estrés durante la infusión de prednisona y L-asparaginasa en la fase de inducción de su debut de leucemia linfoblástica aguda; la madre de la paciente tuvo diabetes gestacional y la abuela y un tío presentaban diabetes mellitus tipo 2. Podríamos especular con una mayor sensibilidad a la exposición a drogas diabetógenas en una paciente predispuesta genéticamente.

¿En qué patologías se presenta la hiperglucemia por estrés con mayor frecuencia?

Puede ser de origen endógeno y asociarse a gastroenteritis, ${ }^{15}$ deshidratación hipernatrémica, ${ }^{24}$ fiebre elevada mayor de $39^{\circ} \mathrm{C}$, asociada a dolor por trauma o convulsiones febriles ${ }^{21}$ en sepsis grave y pacientes críticos en cuidados intensivos, ${ }^{5}$ enfermedades respiratorias, ${ }^{9}$ traumatismo encéfalo-craneano grave, en el que empeora el pronóstico. ${ }^{6}$

También puede ser de origen exógeno, en casos de uso de drogas en pacientes con enfermedades agudas y que pueden producir hiperglucemia o ser pancreatotóxicas; ejemplo: uso de corticoides y L-asparaginasa en la inducción de la leucemia aguda. ${ }^{3}$

Los pacientes que presentaron hiperglucemia con el uso de L-asparaginasa fueron aquellos con edad mayor de 9,5 años, riesgo de obesidad, antecedentes familiares de diabetes mellitus y tratamiento concomitante con corticoides..$^{16,17}$

\section{CONCLUSIÓN}

Frente a un paciente con hiperglucemia en el DEP, debemos descartar diabetes mellitus de debut, ya sea descompensada y con cetoacidosis o compensada, el hallazgo de una hiperglucemia casual en un paciente obeso y con diabetes tipo 2, la hiperglucemia sin descompensación de una diabetes monogénica o ser una hiperglucemia por estrés.

Estas últimas suelen ser no cetósicas, ${ }^{4,9}$ limitadas a la enfermedad aguda y, generalmente, no desarrollan diabetes en el seguimiento posterior. El uso de insulina en los casos más graves $^{5}$ o hipoglucemiantes orales en adultos con hiperglucemia por estrés mejoran la evolución y disminuyen la morbimortalidad. ${ }^{29}$

Existe una superposición de cuadros intermedios en la presentación de la hiperglucemia en el DEP, que requiere el auxilio del especialista para desentrañar el cuadro subyacente.

\section{REFERENCIAS}

1. Alpern ER, Stanley RM, Gorelick MH, Donaldson A, et al. Epidemiology of a pediatric emergency medicine research network: the PECARN Core Data Project. Pediatr Emerg Care 2006;22(10):689-99.

2. Bilkis MD, Vásquez M, González Favre C, Vasicek M, et al. Estudio multicéntrico de la urgencia pediátrica. Arch Argent Pediatr 2006;104(4):301-8.

3. Sament S, Schwartz MB. Severe diabetic stupor without ketosis. S Afr Med J 1957;31(36):893-4.

4. BilkisMD, TraynorV.Hiperglucemia sin cetosis en leucemia linfoblástica aguda. Arch Argent Pediatr 1996;94(1):46-8.

5. Poddar B. Treating hyperglycemia in the critically ill children: is there enough evidence? Indian Pediatr 2011;48(7):531-6.

6. Aşılığlu N, Turna F, Paksu MS. Admission hyperglycemia is a reliable outcome predictor in children with severe traumatic brain injury. J Pediatr (Rio J) 2011;87(4):325-8.

7. Dungan KM, Braithwaite SS, Preiser JC. Stress hyperglycaemia. Lancet 2009;373(9677):1798-807.

8. Bilkis MD, Bou-Khair M, Labaronnie H, Onaindia J. Malrotación intestinal con vólvulo, asociada a hiperglucemia grave por estrés. Arch Argent Pediatr 2011;109(6):e122-5.

9. Weiss SL, Alexander J, Agus MS. Extreme stress hyperglycemia during acute illness in a pediatric emergency department. Pediatr Emerg Care 2010;26(9):626-32.

10. Trifone L. Cetoacidosis diabética. En: Asociación de Profesionales del Hospital de Niños Ricardo Gutiérrez, ed. Guía de Atención en la Urgencia Pediátrica. Buenos Aires; 2005. Págs. 83-5.

11. Castaño L, Bilbao JR, Rica I. Etiopatogenia de la diabetes tipo 1. En: Barrio Castellanos R, Pavía Sesma C, Yturriaga Matarranz R, eds. Diabetes mellitusen la infancia yadolescencia. Madrid: Ediciones Díaz de Santos; 1997. Págs.11-23.

12. Oron T, Gat-Yablovski G, Lazar L, Phillip M, et al. Stress hyperglycemia: a sign of familial diabetes in children. Pediatrics 2011;128(6):e1614-7.

13. Varadarajan P, Sangaralingam T, Senniappan S, Jahnavi S, et al. Clinical profile and outcome of infantile onset diabetes mellitus in southern India. Indian Pediatr 2013;50(8):759-63.

14. Kochar IP. Infantile onset diabetes mellitus. Indian Pediatr 2013;50(8):737.

15. Rabinowitz L, Joffe BI, Abkiewitz C, Shires R, et al. Hyperglycaemia in infantile gastroenteritis. Arch Dis Child 1984;59(8):771-5.

16. Palma RP, Folatre BI, Kyonen LM, Cea SG, et al. Hiperglucemia en niños con leucemia linfoblástica aguda en tratamiento con L-asparaginasa. Rev Chil Pediatr 2013;84(4):387-95.

17. Roberson JR, Raju S, Shelso J, Pui CH, et al. Diabetic ketoacidosisduringtherapy forpediatricacutelymphoblastic leukemia. Pediatr Blood Cancer 2008;50(6):1207-12.

18. Macrae D, Grieve R, Allen E, SadiqueZ, et al. A randomized trial of hyperglycemic control in pediatric intensive care. N Engl J Med 2014;370(2):107-18.

19. Agus MS. Tight glycemic control in children - is the target in sight? N Engl J Med 2014;370(2):168-9.

20. Vardi P, Shehade N, Etzioni A, Herskovits T, et al. Stress hyperglycemia in childhood: a very high risk group for the development of type I diabetes. J Pediatr 1990;117(1 Pt 1):75-7.

21. Shehadeh N, On A, Kessel I, Perlman R, et al. Stress hyperglycemia and the risk for the development of type 1 diabetes. J Pediatr Endocrinol Metab 1997;10(3):283-6.

22. BhisitkulDM, Vinik AI,Morrow AL, SheJX, etal.Prediabetic 
markers in children with stress hyperglycemia. Arch Pediatr Adolesc Med 1996;150(9):936-41.

23. Valerio G, Franzese A, Carlin E, Pecile P, et al. High prevalence of stress hyperglycaemia in children with febrile seizures and traumaticinjuries. Acta Paediatr 2001;90(6):61822.

24. Bordbar MR, Taj-Aldini R, Karamizadeh Z, Haghpanah $S$, et al. Thyroid function and stress hormones in children with stress hyperglycemia. Endocrine 2012;42(3):653-7.

25. Rosenbloom AL, Hunt SS. Prognosis of imparied glucose tolerance in children with stress hyperglycemia, symptoms of hypoglycemia, or asymptomatic glucosuria. J Pediatr 1982;101(3):340-4.
26. Saz EU, Ozen S, Simsek Goksen D, Darcan S. Stress hyperglycemia in febrile children: relationship to prediabetes. Minerva Endocrinol 2011;36(2):99-105.

27. Latorre HA, Drash A. Stress hyperglycemia re development of diabetes. J Pediatr 1991;118(5):827-8.

28. Heggarty H, Trindade H, Bryan EM. Hyperglycaemia in hyperosmolar dehydration. Arch Dis Child 1973;48(9):740-1.

29. Gore DC, Wolf SE, Herndon DN, Wolfe RR. Metformin blunts stress-induced hyperglycemia after thermal injury. J Trauma 2003;54(3):555-61.

30. American Diabetes Association. Diagnosis and classification of diabetes mellitus. Diabetes Care 2013;36 Suppl 1:67-74.

\section{Fe de erratas}

En el listado de los Revisores de los trabajos recibidos durante el año 2014, publicado en Arch Argent Pediatr 2014; 112(6):583-584, se omitió mencionar al Dr. Carlos Grandi, quien se desempeñó como revisor en ese período. 